\title{
Optimizing the Mixing Proportion with Neural Networks Based on Genetic Algorithms for Recycled Aggregate Concrete
}

\author{
Sangyong Kim, ${ }^{1}$ Hee-Bok Choi, ${ }^{2}$ Yoonseok Shin, ${ }^{3}$ Gwang-Hee Kim, ${ }^{3}$ and Deok-Seok Seo ${ }^{4}$ \\ ${ }^{1}$ School of Construction Management and Engineering, University of Reading, Reading RG6 6AW, UK \\ ${ }^{2}$ Department of Architectural Engineering, Jeju National University, Jeju 690-756, Republic of Korea \\ ${ }^{3}$ Department of Plant \& Architectural Engineering, Kyonggi University, Gwanggyosan-Ro, Yeongtong-Gu, Suwon-Si, \\ Gyeonggi-Do 443-760, Republic of Korea \\ ${ }^{4}$ Department of Architectural Engineering, Halla University, Wonju-Si 220-712, Republic of Korea
}

Correspondence should be addressed to Gwang-Hee Kim; ghkim@kyonggi.ac.kr

Received 1 May 2013; Accepted 8 July 2013

Academic Editor: Alex Li

Copyright (C) 2013 Sangyong Kim et al. This is an open access article distributed under the Creative Commons Attribution License, which permits unrestricted use, distribution, and reproduction in any medium, provided the original work is properly cited.

This research aims to optimize the mixing proportion of recycled aggregate concrete (RAC) using neural networks (NNs) based on genetic algorithms (GAs) for increasing the use of recycled aggregate (RA). NN and GA were used to predict the compressive strength of the concrete at 28 days. And sensitivity analysis of the NN based on GA was used to find the mixing ratio of RAC. The mixing criteria for RAC were determined and the replacement ratio of RAs was identified. This research reveal that the proposed method, which is NN based on GA, is proper for optimizing appropriate mixing proportion of RAC. Also, this method would help the construction engineers to utilize the recycled aggregate and reduce the concrete waste in construction process.

\section{Introduction}

Recycled aggregate concrete (RAC) has been widely studied in Korea as part of the effort to preserve natural resources and prevent environmental disruption. Currently, many researchers have studied about application of recycled aggregates (RAs) as the base or subbase material in road construction [1] and as a component of high-strength/highperformance concrete [2]. However, RAs have an unavoidable major defect, that is, microcracks and impurities which, occur in the crushing process of original concrete to produce RAs. These microcracks and impurities lead to RAs with higher water absorption, lower specific gravity, and lower durability than natural aggregates (NAs) [3-5]. These RAs have influence on the properties of concrete, such as their workability, shrinkage, tensile strength, compressive strength, and durability in fresh and hardened concrete states.

Therefore, it should be more effective to determine the mixing proportion for RAC than to attempt to improve the quality of the RAs. The quality of concrete, as determined by its compressive strength and durability, depends on the mixing proportions of the concrete and the mixing preparation technique, as well as on the quality of the concrete components [6]. But to date, the mixing proportions of RAC have been determined by experience and knowledge of previous research based on NAs [6]. However, it is inappropriate to determine the mixing proportions of RAC by this way because of the properties of RAs (high water absorption, low specific gravity, and microcracking). Because interrelationships between RAs and the other components of concrete are complex, old cement mortar that does not hydrate on the surface of RAs when attached much, and when using the RAs, this material progresses hydration with reactions with water again. This case, heat of hydration increases because cement amount that reacts actually becomes more than the designed cement amount. Also, a compression, tension, shearing strength, and so forth of RAC should be bigger than normal concrete by augmented cement content theoretically, but actuality is not so, as their interactions are difficult to express by a mathematical (mathematical formula) model $[7,8]$ and will consume a lot of time. That is, a quantity of all mixing elements should be crystallized again 
by an amount of old cement mortar that does not perform hydration reaction when attached on the surface of RAs.

Therefore, it applied a neural network (NN) and a genetic algorithm (GA) to the mixing of RAC as a tool for the solution of those problems. This research proposes criteria for optimal mixing design of a RAC by sensitivity analysis of NN. Also, with designed mixing proportion, it is able to estimate the compressive strength of RAC. Changes in the quality of the RAC according to the mixing ratio of its components were verified by experimental research in the laboratory, and the quality of RAC predicted by the applied NN was compared with the experimental data.

\section{Methodology}

This research was divided into three main phases (Figure 1). The first step is the processes that make a new data of a variety of kinds for applying to NN-GA model. The second step is the construction of NN model. The third step is applying GA to constructed $\mathrm{NN}$ for running of the optimum of $\mathrm{NN}$, and then applying the new data made in first step to the optimized NN. And sensitivity analysis was performed in the constructed NN-GA model.

Generally, the NN is designed for the specific set of input as well as output. The number of inputs and outputs is not restricted, which is one advantage of $\mathrm{NN}[9,10]$. Then, the number of hidden layer and the number of hidden node are defined. But a large amount of time must be spent in determining them, which is one drawback of $\mathrm{NN}$, because it actually requires some trial-and-error process [1113]. Therefore, to pare down trifling by repeat of trial-anderror process [14] and to systematic access method [15], the GA was applied to the NN to optimize each parameter of the NN (Figure 1).

\section{Experimental Design}

3.1. Component Materials. The constituents of the concrete used in this study included ASTM C 150 Type I Portland cement, the specific gravity of which is 3.16 , recycled coarse aggregates (RCA), and recycled fine aggregates (RFA), made using first-class aggregates produced from a RAs manufacturing corporation, which is BLUESTONE Corporation in South Korea (water absorption ratios: RFA $\leq 5 \%$, RCA $\leq 3 \%$; specific gravity: RFA $\geq 2.2$, RCA $\geq 2$.2-KS F 2527, 2574; Korea Standard). The physical properties of the NAs and RAs are shown in Table 1. When it used such RAs because impurities stick much on its surface by production process, compressive strength of RAC decreased $[16,17]$. Therefore, in this research, the used RAs were washed in water to remove the effect of impurities on concrete strength. RAs that were wash in water were used after uncover for 48 hours in air.

3.2. Mixing of Concrete. The American Concrete Institute (ACI) Standard 211.1, "Recommended Practice for Selecting Proportions for Normal-Weight Concrete," was used to proportion the concrete mixtures. The RFA was replaced at $0 \%$, $10 \%, 30 \%, 50 \%, 70 \%$, and $100 \%$ (by weight), and the RCA was replaced at $0 \%, 30 \%, 50 \%$, and $100 \%$ (by weight). Table 2 shows a sample of the specified concrete mixing proportions in terms of the replacing ratios for RCA and RFA. The data in Table 2 is used to train the neural networks.

Admixtures, unit water content, unit cement content, and designed compressive strength are as follows:

(i) water/cement ratio (W/C): 50\%;

(ii) unit water content: $175 \mathrm{~kg} / \mathrm{m}^{3}$;

(iii) unit cement content: $350 \mathrm{~kg} / \mathrm{m}^{3}$;

(iv) designed compressive strength: $35 \mathrm{MPa}$.

All test procedures in sieve analysis, specific gravities, and the absorptions of aggregates conformed to ASTM Standards C 136, C 127, and C 128, respectively. Making and curing the concrete and the compressive strength of the cylindrical concrete specimens conformed to ASTM C 192 and ASTM C 39 , respectively.

3.3. Casting Test Specimens. Seventy-two casts, for each of which five cylindrical (28-day) concrete specimens were cast (i.e., a total of 360), were prepared for mechanical testing. A cylindrical concrete specimen with a diameter of $100 \mathrm{~mm}$ and a depth of $200 \mathrm{~mm}(\phi 100 \times 200 \mathrm{~mm})$ was used to test the compressive strengths of the samples of RAC. The specimens were casted after the initial curing for 24 hours. Curing was maintained for 28 days in constant temperature and humidity chamber, and the uniaxial compressive test was performed on day 28 by UTM (Universal Testing Machine). The slump of the fresh concrete and the air content were also measured. In Table 2, each data point for slump value and air content rate represents a single test result, and the compressive strength value represents the average value of three test results, after the highest and lowest values among five tests were excluded. To train and test the NN, 216 data sets were used.

3.4. Verifying the Experimental Results. Figure 2 shows a histogram of compressive strength, which is the result of description statistics about experimental data by statistical package for social science. The test results for the cylindrical concrete specimens show a normal distribution, with a mean of $33.75 \mathrm{MPa}$ and a standard deviation of $2.82 \mathrm{MPa}$. The range of strengths obtained by testing agreed well with the range of the normal distribution curve according to ACI 214-77 [18]. Up to this point in the research, the authors used the mixing proportions for normal concrete as the mixing proportions for the RAC to obtain compressive strength data for the RAC. An average RAC strength was achieved when the mixing proportions of normal concrete with strength of $35 \mathrm{MPa}$ were used for the RAC. To achieve a suitable slump and air content that would allow effective workability of the RAC under the conditions of this experiment, the proper design strength for the RAC should be 33 to $34 \mathrm{MPa}$. Therefore, as described in Section 4 , the range of output values used for the strength estimation and sensitivity analysis of RAC performed in this research was chosen to be 33 to $34 \mathrm{MPa}$. 


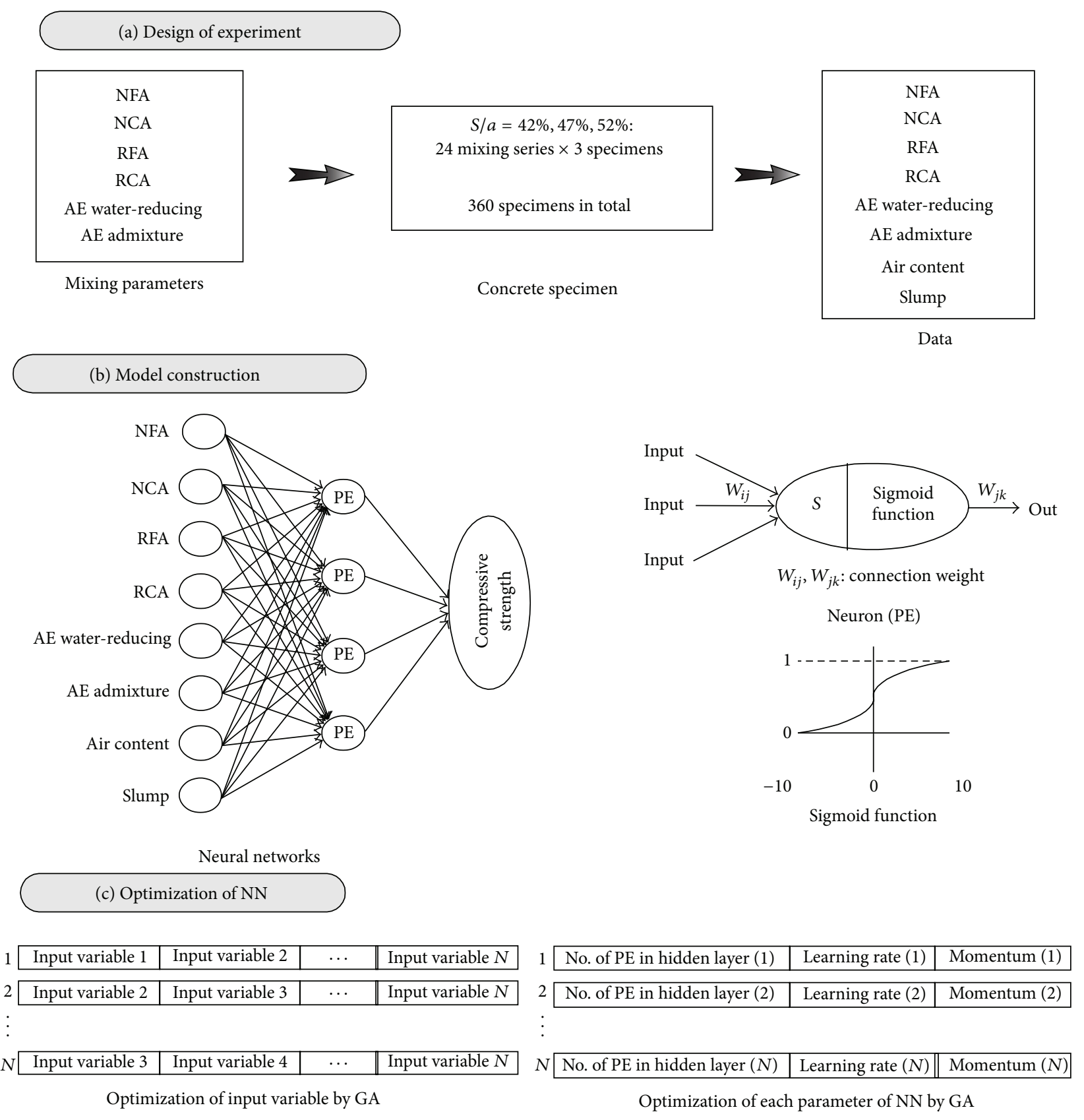

FIgURE 1: The three steps of this study for RAC mixing. (a) Each factor and S/a value of NAs and RAs were used to construct the NN model using how they satisfied the concrete performance criteria (e.g., slump, air content, admixture, and compressive strength), and how they matched the selected factor to receive the factor's optimum value. (b) Each factor's value obtained in " $a$ " was applied to the NN input and output variables. The neuron that received the input value displayed the output value using a connection weight. This time and connection weight were calculated using a sigmoid transfer function. (c) The GA was applied to optimize each NN parameter (e.g., momentum, learning rate of the $\mathrm{NN}$, and number of neurons in the hidden layers).

\section{Model Construction}

In this section, the construction of a compressive strength prediction model based on NN and GA is described. The NN architecture was composed of three layers (Figure 3): input layer, hidden layer, and output layer. The input parameters are seven: NFA, NCA, RFA, RCA, AE water-reducing, AE admixture content, air content, and slump. The output parameter is the compressive strength at day 28. A sample of the cases (24 cases of each S/a: $42 \%, 47 \%$, and 52\%) that were used in the NN training is listed in Table 2.

The learning of $\mathrm{NN}$ is accomplished by a backpropagation algorithm (BPN), and the BPN has one of the following transfer functions sigmoid, linear, and exponential functions, that are used to calculate the output for each neuron, except for the input neuron. Among those transfer functions, sigmoid 
TABLE 1: Physical properties of RAs (1st class) and NAs.

\begin{tabular}{lccc}
\hline $\begin{array}{l}\text { Source of } \\
\text { aggregate }\end{array}$ & $\begin{array}{l}\text { Fineness } \\
\text { modulus }\end{array}$ & $\begin{array}{c}\text { Specific } \\
\text { gravity }\end{array}$ & $\begin{array}{c}\text { Water } \\
\text { absorption } \\
(\%)\end{array}$ \\
\hline NCA & 6.52 & 2.64 & 1.24 \\
NFA & 2.52 & 2.55 & 1.53 \\
RCA & 6.65 & 2.53 & 2.86 \\
RFA & 3.89 & 2.43 & 4.95 \\
\hline
\end{tabular}

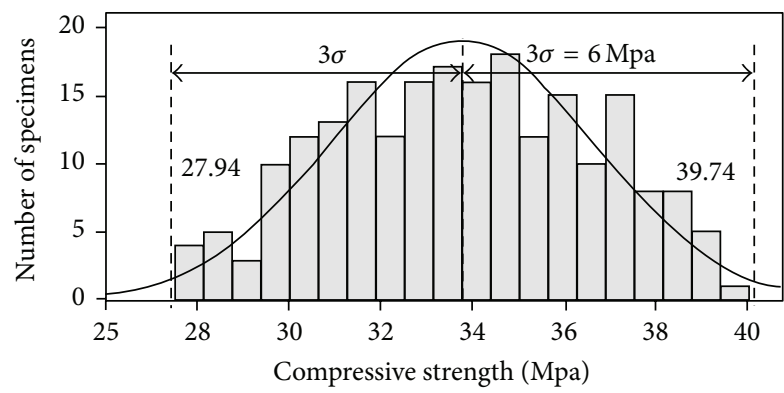

Mean $=33.746$

Std. Dev. $=2.82297$

$N=216$

FIGURE 2: Histogram of compressive strengths of $S / a$ : $42 \%, 47 \%$, and $52 \%$.

function is used most extensively and has many advantages:

(1) has two advantages of linear and exponential functions,

(2) is nonlinear function, (3) is differentiable function, and

(4) is a S-Pattern. Therefore, the sigmoid function was used as transfer function in this study.

4.1. NN and GA. The NN is inspired by the neuronal structure and operation of the biological brain. Figure 3 shows the simple architecture of backpropagation network with a threelayer network that was used in this study, which consists of an input layer, a hidden layer, an output layer, and the connections between them. The learning mechanism of this backpropagation network is a generalized delta rule, which performs a gradient descent on the error space to minimize the total error between the calculated and desired values of the output layer during modification of the connection weights. The detailed process about backpropagation network is as follows.

(i) At early, $\mathrm{NN}$ does connection weights $W_{j i}$ and $W_{k j}$ and biases $\theta_{j}$ and $\theta_{k}$ value to give randomly. Input value $N_{j}$ and activated value $N_{j}$ of hidden layer neuron are calculated as a result value $O_{k}$ for forward by the following equation:

$$
\begin{gathered}
N_{j}=W_{j i} I_{i}+\theta_{j}, \\
H_{i}=f\left(N_{j}\right) .
\end{gathered}
$$

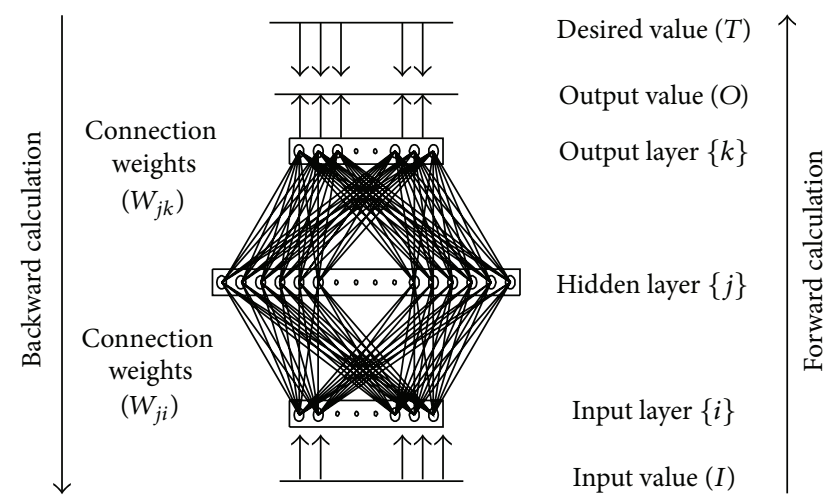

FIGURE 3: Backpropagation of NN.

Here, $f(x)$ is activation function (sigmoid function), and sigmoid function is represented by the following equation:

$$
f(x)=\frac{1}{1+e^{-\left(\sum x w-\theta_{i}\right)}} .
$$

(ii) Delta $(\delta)$ is calculated by (3) as difference of target values $(D)$ and result values $(O)$ :

$$
\delta=(D-O) \times O(I-O)
$$

(iii) NN calculates for backward from output layer again. Do a connection weights $(W)$ that is inputted in output layer which is adjusted by the $\Delta W$ of the following equation:

$$
\begin{gathered}
W_{\text {new }}=W_{\text {old }}+\Delta W, \\
\Delta W=\eta \delta H,
\end{gathered}
$$

where $\eta$ is in the range of between 0 and 1 as invariable of leaning ratio.

(iv) Also, it calculates for backward from hidden layer again. The hidden layer, by the same formula that is calculated for backward in output layer, is calculated toward input layer from the nearest layer to output layer, and the delta of output layer is as follows:

$$
\delta=\left(\sum \delta W\right) \times O(I-O) .
$$

Here, the connection weighting of inputted hidden layer is adjusted by equal method at (iii) step. This backward process is calculated through iteration until it reaches a global minimum point.

(v) Repeat all learning data from (i) to (iv) steps. Uniting MSE (mean squared error) value of all learning data that get by repeating, learning of once is completed

$$
\text { MSE }=\frac{1}{2 P} \sum_{j=1}^{p} \sum_{i=1}^{n}\left(D_{i j}-O_{i j}\right)^{2} .
$$

In (6), $n$ is the number of hidden layer neurons, and $p$ is the number of learning data.

(vi) If the MSE is not satisfied target error value from (i) step and reached in target error value, this circulation operation is repeated continuously. 
TABLE 2: Sample of specified concrete mixing proportions used for NN training.

\begin{tabular}{|c|c|c|c|c|c|c|c|c|c|c|c|}
\hline \multirow{2}{*}{ S/a (\%) } & \multicolumn{4}{|c|}{ Unit content $\left(\mathrm{kg} / \mathrm{m}^{3}\right)$} & \multicolumn{2}{|c|}{ Admixtures (g) } & \multirow{2}{*}{ Air (\%) } & \multirow{2}{*}{ Slump $(\mathrm{cm})$} & \multirow{2}{*}{\multicolumn{3}{|c|}{ Compressive strength (MPa) }} \\
\hline & NCA & RCA & NFA & RFA & AE-reducing & AE agent & & & & & \\
\hline \multirow{8}{*}{42} & 1008.68 & 0 & 724.81 & 0 & 196 & 4.9 & 4.3 & 21.5 & 33.71 & 33.79 & 33.87 \\
\hline & 1008.68 & 0 & 652.33 & 72.48 & 196 & 4.9 & 3.9 & 22 & 30.45 & 28.43 & 30.43 \\
\hline & 706.08 & 302.60 & 507.37 & 217.44 & 196 & 2.45 & 5.8 & 22 & 33.64 & 32.65 & 33.74 \\
\hline & 706.08 & 302.60 & 362.405 & 361.405 & 196 & 2.45 & 2.0 & 23 & 35.79 & 32.80 & 37.02 \\
\hline & 504.34 & 504.34 & 362.405 & 361.405 & 196 & 2.45 & 2.8 & 25 & 33.71 & 36.13 & 34.38 \\
\hline & 504.34 & 504.34 & 0 & 724.81 & 196 & 2.45 & 2.3 & 20 & 31.67 & 31.68 & 26.35 \\
\hline & 0 & 1008.68 & 724.81 & 0 & 196 & 2.45 & 4.5 & 17 & 32.22 & 28.39 & 28.58 \\
\hline & 0 & 1008.68 & 652.33 & 72.48 & 196 & 2.45 & 4.5 & 20 & 30.42 & 28.90 & 32.34 \\
\hline \multirow{8}{*}{47} & 921.73 & 0 & 567.77 & 229.19 & 196 & 1.225 & 6.0 & 22.5 & 35.83 & 36.01 & 35.44 \\
\hline & 921.73 & 0 & 405.55 & 381.97 & 196 & 1.225 & 3.3 & 10 & 38.16 & 38.23 & 36.16 \\
\hline & 645.21 & 270.14 & 729.99 & 76.39 & 196 & 1.225 & 5.6 & 11 & 34.91 & 35.30 & 33.52 \\
\hline & 645.21 & 270.14 & 567.77 & 229.19 & 196 & 1.225 & 4.7 & 17 & 35.94 & 35.03 & 34.66 \\
\hline & 460.86 & 450.23 & 567.77 & 229.19 & 196 & 1.225 & 3.8 & 22.5 & 33.70 & 29.98 & 35.30 \\
\hline & 460.86 & 450.23 & 405.55 & 381.97 & 196 & 1.225 & 3.3 & 19 & 32.35 & 35.48 & 35.23 \\
\hline & 0 & 900.46 & 243.33 & 534.76 & 196 & 1.225 & 2.5 & 21 & 34.15 & 34.11 & 34.92 \\
\hline & 0 & 900.46 & 0 & 763.94 & 196 & 1.225 & 3.5 & 17 & 30.52 & 31.34 & 33.15 \\
\hline \multirow{8}{*}{52} & 834.77 & 0 & 807.64 & 84.52 & 196 & 0 & 7.2 & 22.5 & 29.08 & 30.27 & 29.99 \\
\hline & 834.77 & 0 & 628.17 & 253.26 & 196 & 0 & 6.8 & 23 & 27.64 & 29.29 & 30.61 \\
\hline & 584.34 & 244.65 & 897.38 & 0 & 196 & 0 & 2.1 & 25 & 30.65 & 33.06 & 34.66 \\
\hline & 584.34 & 244.65 & 448.69 & 422.6 & 196 & 0 & 2.8 & 21 & 32.87 & 30.62 & 29.82 \\
\hline & 417.39 & 407.75 & 807.64 & 84.52 & 196 & 0 & 3.4 & 16.5 & 32.78 & 33.13 & 31.87 \\
\hline & 417.39 & 407.75 & 628.17 & 253.26 & 196 & 0 & 3.4 & 16 & 33.09 & 34.03 & 33.48 \\
\hline & 0 & 815.51 & 897.38 & 0 & 196 & 0 & 3.0 & 14.5 & 35.60 & 31.52 & 32.64 \\
\hline & 0 & 815.51 & 807.64 & 84.52 & 196 & 0 & 3.4 & 15 & 35.63 & 29.06 & 29.97 \\
\hline
\end{tabular}

The GA employs Darwinian selection and Mendelian crossover principles. Because GAs are robust and guided random search methods, they have found a niche in the nonlinear programming field. GA is based on the collective learning of a population, the individuals of which represent the potential solutions for the problem to be solved. GA transfers a group of genetic individuals from one generation to the next. A set of individuals from the same generation is known as a population. Each population goes through a series of genetic operators, that is, selection, recombination, or variation, to produce the next generation. An in-depth analysis is given in [19].

$\mathrm{NN}$ is commonly used for difficult tasks involving intuitive judgment or requiring the detection of data patterns that elude conventional analytic techniques. The performance of NN, however, is affected by the network architecture and its parameter settings. In NN models, these factors have been determined by heuristic and trial-and-error methods, which are time-consuming and tedious [14]. Therefore, in this research, a primary role of the GA is to determine the number of neurons in the hidden layers, the momentum, the learning rate of the $\mathrm{NN}$ and to select the appropriate input variables. Application method of GA to search for a combination of input variables of $\mathrm{NN}$ for optimum performance of $\mathrm{NN}$ model is as follows. (i) If the number of input variables is " $n$," GA composes individual by random such as in Figure 1(c) with input variable of " $n$." This time, number of individual is created as population size $(N)$ of GA.

(ii) Each individual corresponded with input variable of $\mathrm{NN}$, and corresponded input variable is applied to $\mathrm{NN}$ and the NN learn. The learned MSE of NN gets fitness value of each chromosome.

(iii) Individuals which the fitness valued pass hybridization (crossover) or mutation process. At this process, individuals that have excellent fitness value exist, and individuals that are not so disappear.

(iv) Until reaching optimum result, process of (ii) and (iii) as established number of households is repeated. Passing through this process, chromosomes that have a bad influence upon result value of NN disappears, and individuals whose fitness is superior exist. As this, combination of input variable whose estimation correctness of NN is high is decided. A more in-depth content is given in [20].

4.2. Implementation of $N N$ and GA. The GA application process for optimizing the number of input variables in the input layer, the number of neurons in the hidden layer, and 


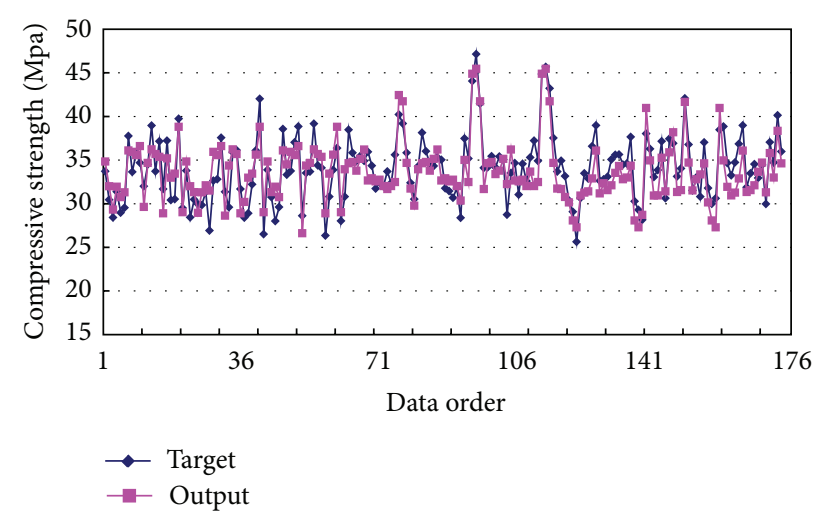

FIGURE 4: Results of training with 176 data sets.

the coefficient of learning rates of NN was as follows. First, the numbers of hidden layers and output neurons in the $\mathrm{NN}$ were set to 1 (2 and 3) and 1, respectively. All chromosomes were automatically set in NN so that they consisted of the numbers of input variables, of hidden neurons, and of learning rates. NN also automatically produced their initial values. The values for these input variables were set in a range with a lower limit of $X$ and an upper limit of $n X$ (where $X$ is an input variable and $n$ is the number of input variables). The hidden neuron number was set in a range with a lower limit of $0.75 \mathrm{~m}$ and an upper limit of $2 m+1$ (where $m$ is the number of hidden neurons). The step size had a lower limit of 0 and an upper limit of 1 , and the momentum had a lower limit of 0 and an upper limit of 1 . The numbers of chromosomes (population size) and generations were set to 100 and 50, respectively.

After the parameter values (number of input variables, hidden neurons, step size, and momentum for each chromosome) were translated into the predefined NN, the network of $\mathrm{NN}$ was trained on the training data set. A cross-validation data set was used to test whether the stopping criteria were satisfied. The training process for the BPN stopped after a maximum of 1,000 epochs or until there was no improvement in mean squared error (MSE) for 1,000 epochs on the crossvalidation data set. The fitness of every chromosome was evaluated by measuring the MSE, which is the estimated result on a cross-validation data set.

The number of data sets used to train the NN was 176, and the average training error was 5.26\% (lowest training error $0.06 \%$, highest training error $9.94 \%$ ). As can be seen in Figure 4, the model was successfully trained. The model gave values that were very close to the actual values and was able to follow the trend of the actual values. On the other hand, the extreme values of the targeted compressive strengths could not be obtained from the model because the model was conservative, and it required more extreme training data to learn the extremes.

The test results given in Figure 5 are compared with the 40 actually measured data sets tested in the trained model. As can be seen in Figure 5, the performance of the model was very good, with an average error of $5.10 \%$ (lowest $0.21 \%$ and highest 9.23\%).

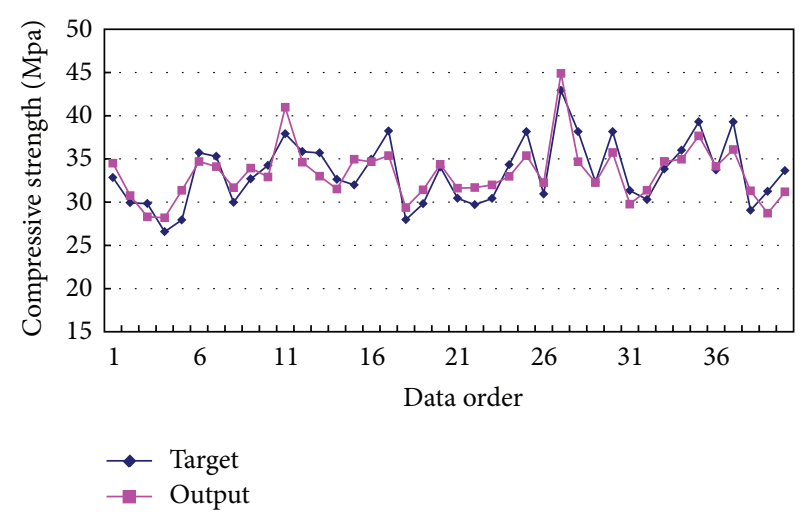

Figure 5: Results of testing the trained model (40 data sets).

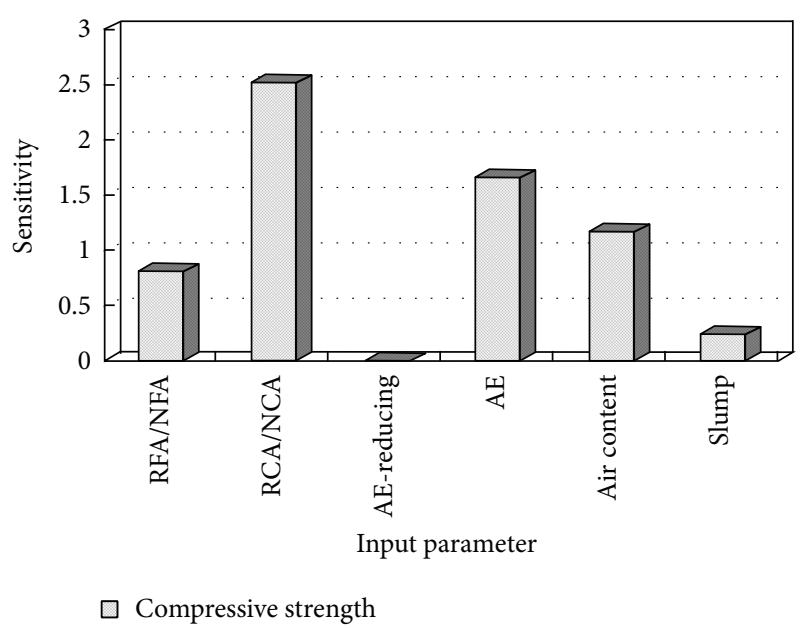

FIGURE 6: Sensitivity about the mean showing the dependence on the input parameters.

4.3. Sensitivity Analysis. The sensitivity of the input variables of the NN (such as RCA, RFA, and air content) to the compressive strength of the RAC was also analysed using the constructed NN model.

Because properties of RAs are different from those of NAs, shape, surface, impurity content, agent usage, and others, it is required to research how much those properties of NAs have an effect on the compressive strength of RAC. And based on the sensitivity analysis, a mixing design of RAC will be made. Sensitivity analysis evaluates the changes in training error resulting from a change in an input value. The 216 data points obtained by experimentation were used to analyse the sensitivity of the input variables. In this study, NN with 2 hidden layers optimized by GA was used for the sensitivity analysis and to map the inputs and outputs.

\section{Result and Discussion}

The sensitivity of RCA/NCA, which represents the change in compressive strength according to the replacement ratio of 


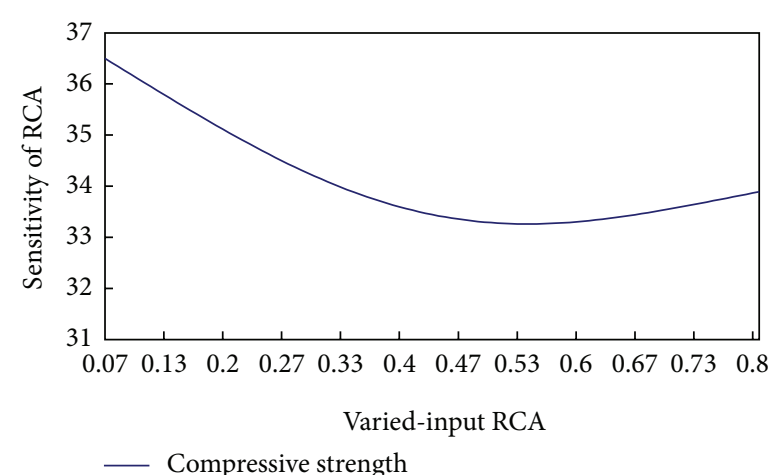

(a)

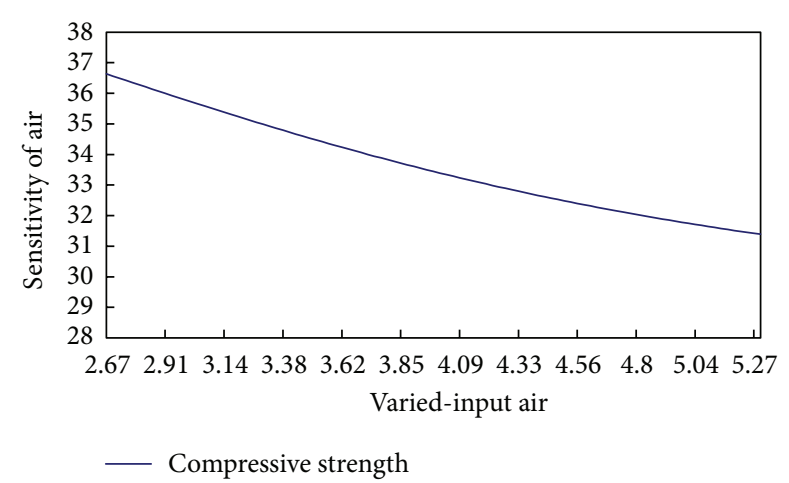

(c)

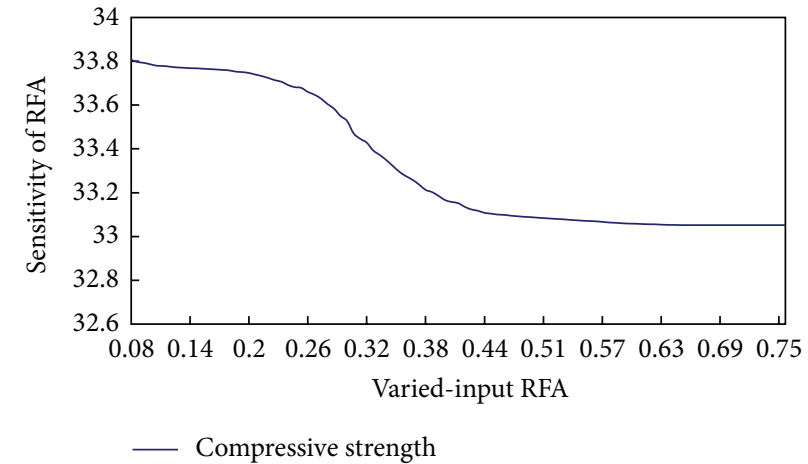

(b)

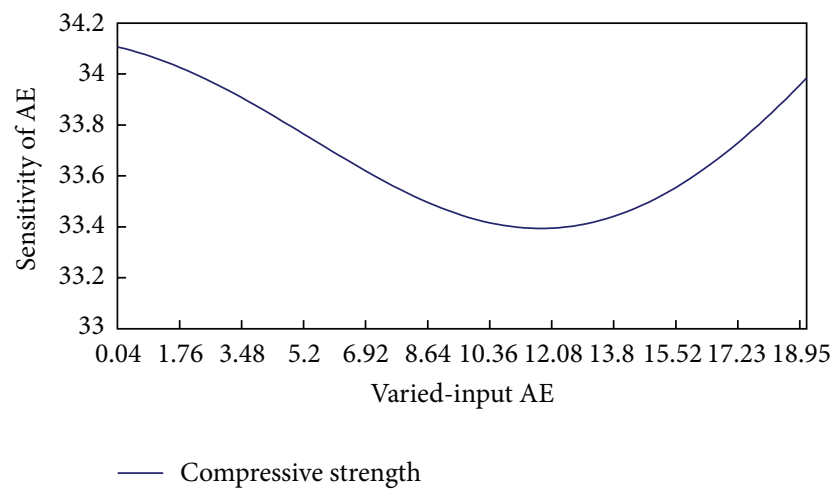

(d)

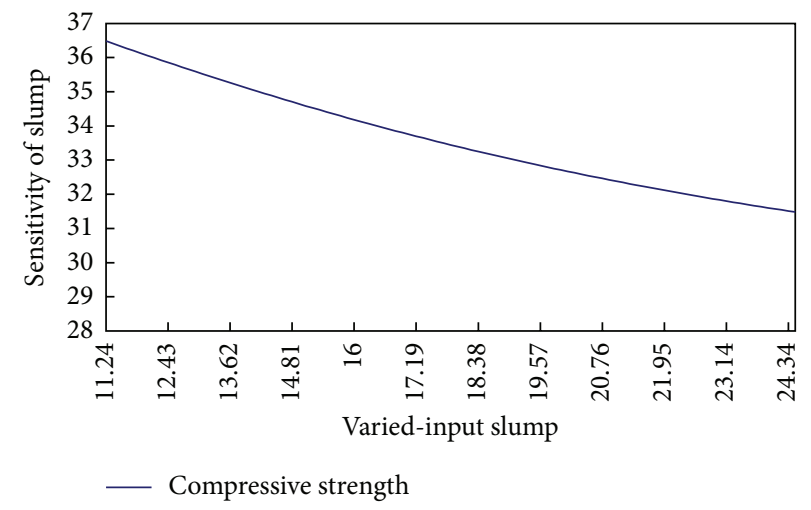

(e)

FIGURE 7: Sensitivity of the input parameters to the compressive strength, showing the effect of varying the input value on the parameters.

RCA, shows a relatively higher value in Figures 6 and 7 than other input parameters because RCA is angular and rough. On the other hand, because the surface area of the bond between an aggregate and cement paste increases or decreases according to changes in the replacement ratio of RCA, the concrete strength can vary sharply. The mixing ratio of RCA affects the compressive strength of RAC.

In Figure 6, the replacement ratio of RFA shows a lower sensitivity value than the replacement ratio for RCA relative to the compressive strength of RAC. And in Figure 7, the compressive strength of RAC decreases with increases in the replacement ratio of RFA. In particular, the compressive strength appears to decrease rapidly between the inputs RFA values of 0.26 and 0.44 . In this case, some water may be absorbed by the RA; the more aggregate, the more water is absorbed. However, an aggregate content above a certain amount would lead to less shrinkage and less bleeding, and therefore, to less damage in the bond between the aggregate and the cement paste [21]. Therefore, whereas the compressive strength was abruptly lost between 0.26 and 0.44 , the compressive strength did not change when the RFA value was greater than 0.44 .

In Figure 7, the output value decreased to 12.08 with increases in $\mathrm{AE}$, and then increased again. This result shows 


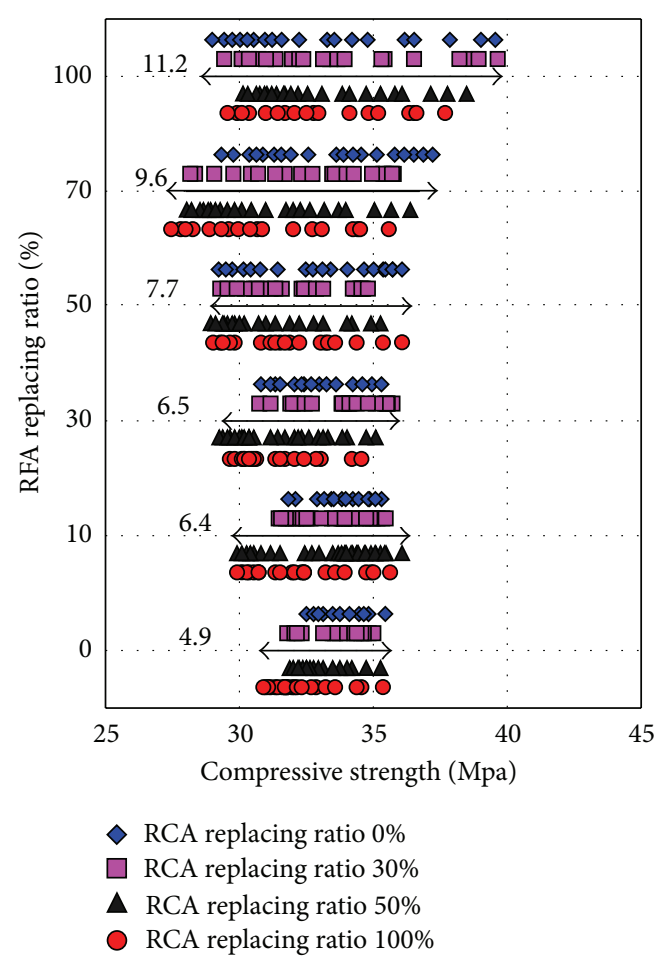

FIgURE 8: Deflection of compressive strength with RCA and RFA replacing ratios.

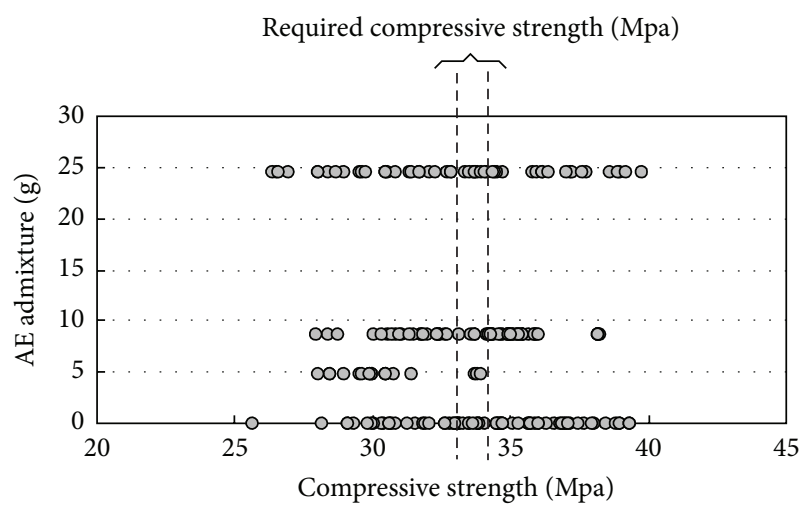

Figure 9: Range of AE admixture.

that the AE admixture acts in RAC in the same way as in normal concrete: when air voids are incorporated into the cement paste matrix, either as a result of inadequate compaction or through the use of an $\mathrm{AE}$ admixture, they also increase the porosity and decrease the strength of the system. The graph of "output $\times$ variable input air" shows that the concrete suffered considerable loss of strength with increasing amounts of entrained air.

Sensitivity analysis shows that compressive strength was more affected by the replacement ratio of RCA than by the replacement ratio of RFA. The deflection of compressive strength by the cross-matches of RCA and RFA is shown in Figure 8. The deflection of compressive strength of RAC becomes more significant as the replacement ratio of RCA

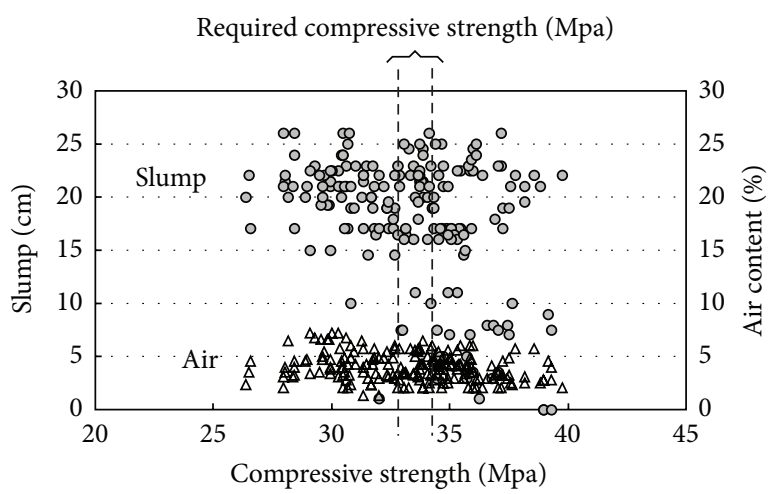

FIGURE 10: Range of slump and air content.

increases. Note that the deflection became very serious when the replacement ratio of RCA exceeded 50\%; with increasing the replacement ratio of RAs, it means that it is difficult to acquire concrete of designed compressive strength. The effects of AE admixture on compressive strength are shown in Figure 9. Under the conditions of this experiment $(\mathrm{W} / \mathrm{C}$ : $50 \%$; unit water content: $175 \mathrm{~kg} / \mathrm{m}^{3}$; unit cement content: $350 \mathrm{~kg} / \mathrm{m}^{3}$; acquired compressive strength: 33 to $34 \mathrm{MPa}$; and S/a: $42 \%, 47 \%$, and $52 \%$ ), the compressive strength of RAC did not change when the amount of AE admixture added to the RAC was between $5 \mathrm{~g}$ and $10 \mathrm{~g}$. However, when the amount of AE admixture was under $5 \mathrm{~g}$ and over $10 \mathrm{~g}$, the compressive strength of the RCA deviated considerably from the average value. Therefore, the AE admixture must be used carefully with the RAC. Slump and air content influence the workability and strength of concrete, although air is used to make concrete resistant to freezing and thawing. The compressive strength of concrete is important, but other properties that are relevant when it is being transported, possibly pumped, and placed, are equally important. The suitable range of slump values and air contents for each is 20 \pm 1.5 and $4.5 \pm 0.5$, respectively (Figure 10 ).

It acquired criteria (Table 3 ) for optimum mixing of RAC that have compressive strength $33 \sim 34 \mathrm{MPa}$ by this research until now. And it makes the RAC confirm whether these criteria are suitable or were not so and measure a slump, an air content, and a compressive strength at 28 days.

The replacement ratio of RAs applied the maximum value (RCA $30 \%$ and RFA $50 \%$ at S/a 42\%; RCA 50\% and RFA $50 \%$ at S/a $47 \%$; RCA $50 \%$ and RFA $30 \%$ at S/a 52\%) that is registered in Table 3 considering the worst situation, and a W/C did the 50\%. There is the result in Table 4 . Air and a slump values among the test values were included in a range of the target value, and average compressive strength of three specimens was lower than the target values except an S/a $42 \%$. But the test values were within the target value in general.

\section{Conclusion}

This study presents an appropriate quality range for RAs and the other components of RAC using sensitivity analysis with neural networks, for use in the production of RAs and 
TABLE 3: Summary of results (approximate range of each parameter).

\begin{tabular}{|c|c|c|c|c|c|c|c|}
\hline \multirow{2}{*}{ Required strength $(\mathrm{MPa})$} & \multirow{2}{*}{ S/a (\%) } & \multirow{2}{*}{ Air (\%) } & \multirow{2}{*}{ Slump $(\mathrm{cm})$} & \multicolumn{2}{|c|}{ Replacement ratio (\%) } & \multicolumn{2}{|l|}{ Admixtures (g) } \\
\hline & & & & RCA (1st Class) & RFA (1st Class) & AE-water reducing agent & AE agent \\
\hline \multirow{3}{*}{$33 \sim 34$} & 42 & & & $0 \sim 30 \%$ & $0 \sim 50 \%$ & 196 & $5 \sim 10$ \\
\hline & 47 & $4.5 \pm 0.5$ & $20 \pm 1.5$ & $0 \sim 50 \%$ & $0 \sim 50 \%$ & 196 & $5 \sim 10$ \\
\hline & 52 & & & $0 \sim 50 \%$ & $0 \sim 30 \%$ & 196 & - \\
\hline
\end{tabular}

TABLE 4: Criteria evaluation.

\begin{tabular}{|c|c|c|c|c|c|c|c|c|c|c|c|c|c|c|}
\hline \multirow{2}{*}{ S/a (\%) } & \multicolumn{6}{|c|}{ Unit content $\left(\mathrm{kg} / \mathrm{m}^{3}\right)$} & \multicolumn{2}{|c|}{ Admixtures (g) } & \multicolumn{3}{|c|}{ Target value } & \multicolumn{3}{|c|}{ Test value } \\
\hline & Cement & Water & NCA & RCA & NFA & RFA & $\begin{array}{l}\mathrm{AE} \text {-water } \\
\text { reducing agent }\end{array}$ & $\begin{array}{c}\mathrm{AE} \\
\text { agent }\end{array}$ & $\begin{array}{l}\text { Air } \\
(\%)\end{array}$ & $\begin{array}{l}\text { Slump } \\
(\mathrm{cm})\end{array}$ & $\begin{array}{c}\text { Strength } \\
\text { (MPa) }\end{array}$ & $\begin{array}{l}\text { Air } \\
(\%)\end{array}$ & $\begin{array}{l}\text { Slump } \\
(\mathrm{cm})\end{array}$ & $\begin{array}{c}\text { Strength } \\
\text { (MPa) }\end{array}$ \\
\hline 42 & & & 719.7 & 291 & 368.9 & 350.2 & 202 & 4.9 & & & & 4.2 & 18.4 & 34.4 \\
\hline 47 & 350 & 175 & 469.7 & 443.1 & 412.9 & 391.9 & 205 & 1.225 & $4.5 \pm 0.5$ & $20 \pm 1.5$ & 33 & 4.1 & 21.7 & 31.7 \\
\hline 52 & & & 425.4 & 401.3 & 632.9 & 257.5 & 203 & 1.014 & & & & 4.2 & 20.1 & 31.5 \\
\hline
\end{tabular}

RAC, and ultimately to promote the use of RAs in concrete. In this study, the mixing criteria of the basic concrete for RAC were determined. RCA content and the AE admixture content are the most important to the compressive strength of RAC. Finally, the results of this study will be applied to use in various mixing proportions of recycled concrete. This research will contribute to improve the usage of the recycled aggregate in construction industry and to reduce the waste in construction process.

\section{References}

[1] T. Bennert, J. Papp W.J., A. Maher, and N. Gucunski, "Utilization of construction and demolition debris under traffic-type loading in base and subbase applications," Transportation Research Record, no. 1714, pp. 33-39, 2000.

[2] A. Ajdukiewiez and A. Kliszezewiez, "Influence of recycled aggregates on mechanical characteristics of HS/HPS," Cement and Concrete Research, vol. 24, no. 2, pp. 269-279, 2002.

[3] Y. H. Lin, Y. Y. Tyan, T. P. Chang, and C. Y. Chang, "An assessment of optimal mixture for concrete made with recycled concrete aggregates," Cement and Concrete Research, vol. 34, no. 8, pp. 1373-1380, 2004.

[4] R. Zaharieva, F. Buyle-Bodin, and E. Wirquin, "Frost resistance of recycled aggregate concrete," Cement and Concrete Research, vol. 34, no. 10, pp. 1927-1932, 2004.

[5] R. M. Salem, E. G. Burdette, and N. M. Jackson, "Resistance to freezing and thawing of recycled aggregate concrete," ACI Materials Journal, vol. 100, no. 3, pp. 216-221, 2003.

[6] K. H. Yang and K. H. Lee, “Tests on alkali-activated slag foamed concrete with various water-binder ratios and substitution levels of fly ash," Journal of Building Construction and Planning Research, vol. 1, no. 1, pp. 8-14, 2013.

[7] J. W. Oh, I. W. Lee, J. T. Kim, and G. W. Lee, "Application of neural networks for proportioning of concrete mixes," $A C I$ Materials Journal, vol. 96, no. 1, pp. 61-67, 1999.

[8] N. Al-Mutairi, M. Terro, and A. Al-Khaleefi, "Effect of recycling hospital ash on the compressive properties of concrete: statistical assessment and predicting model," Building and Environment, vol. 39, no. 5, pp. 557-566, 2004.

[9] G. H. Kim, J. M. Shin, S. Kim, and Y. Shin, "Comparison of school building construction costs estimation methods using regression analysis, neural network, and support vector machine," Journal of Building Construction and Planning Research, vol. 1, no. 1, pp. 1-7, 2013.

[10] J. M. de la Garza and K. G. Rouhana, "Neural networks versus parameter-based applications in cost estimating," Cost Engineering, vol. 37, no. 2, pp. 14-18, 1995.

[11] A. E. Smith and A. K. Mason, "Cost estimation predictive modeling: regression versus neural network," Engineering Economist, vol. 42 , no. 2, pp. 137-161, 1997.

[12] S. Kim, "Hybrid forecasting system based on case-based reasoning and analytic hierarchy process for cost estimation," Journal of Civil Engineering and Management, vol. 19, no. 1, pp. 86-96, 2013.

[13] H. Li, "Neural networks for construction cost estimation," Building Research and Information, vol. 23, no. 5, pp. 279-284, 1995.

[14] T. Hegazy, P. Fazio, and O. Moselhi, "Developing practical neural network applications using back-propagation," Microcomputers in Civil Engineering, vol. 9, no. 2, pp. 145-159, 1994.

[15] E. Vonk, L. C. Jain, and R. P. Johnson, Automatic Generation of Neural Network Architecture Using Evolutionary Computation, World Scientific, Singapore, 1999.

[16] A. Katz, "Properties of concrete made with recycled aggregate from partially hydrated old concrete," Cement and Concrete Research, vol. 33, no. 5, pp. 703-711, 2003.

[17] H. Chen, T. Yen, and K. Chen, "Use of building rubbles as recycled aggregates," Cement and Concrete Research, vol. 33, no. 1, pp. 125-132, 2003.

[18] "ACI 214-77 (Reapproved 1989). Recommended practice for evaluation of strength test results of concrete, ACI Manual of concrete Practice, Part 2: construction Practices and Inspection Pavement," Detroit, Michigan, 1994.

[19] T. Munakata, Fundamentals of the New Artificial Intelligence: Beyond Traditional Paradigms, Springer, New York, NY, USA, 1998. 
[20] G. H. Kim, J. E. Yoon, S. H. An, H. H. Cho, and K. I. Kang, "Neural network model incorporating a genetic algorithm in estimating construction costs," Building and Environment, vol. 39, no. 11, pp. 1333-1340, 2004.

[21] O. Pastor-Bárcenas, E. Soria-Olivas, J. D. Martín-Guerrero, G. Camps-Valls, J. L. Carrasco-Rodríguez, and S. Del Valle-Tascón, "Unbiased sensitivity analysis and pruning techniques in neural networks for surface ozone modelling," Ecological Modelling, vol. 182, no. 2, pp. 149-158, 2005. 

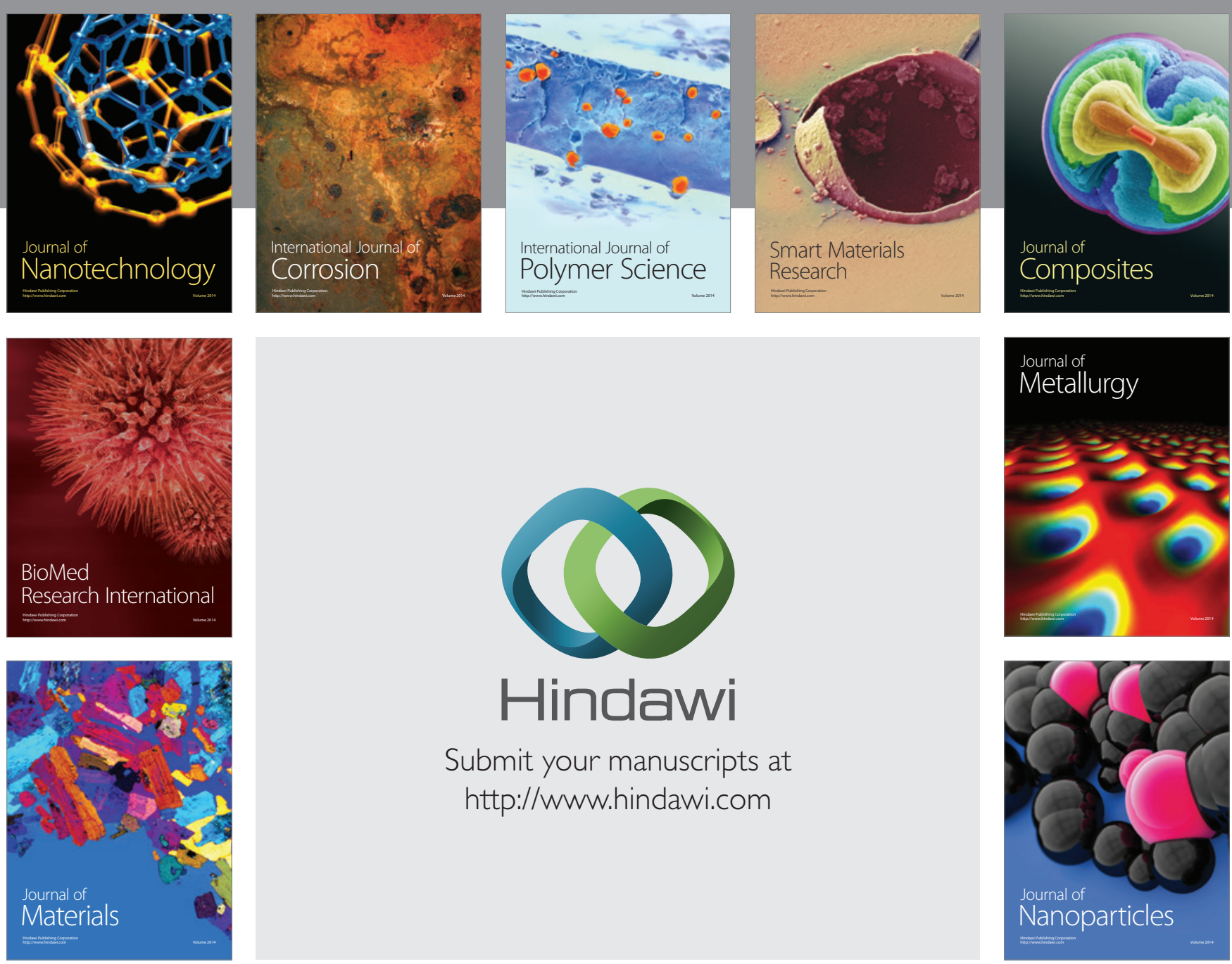

Submit your manuscripts at http://www.hindawi.com
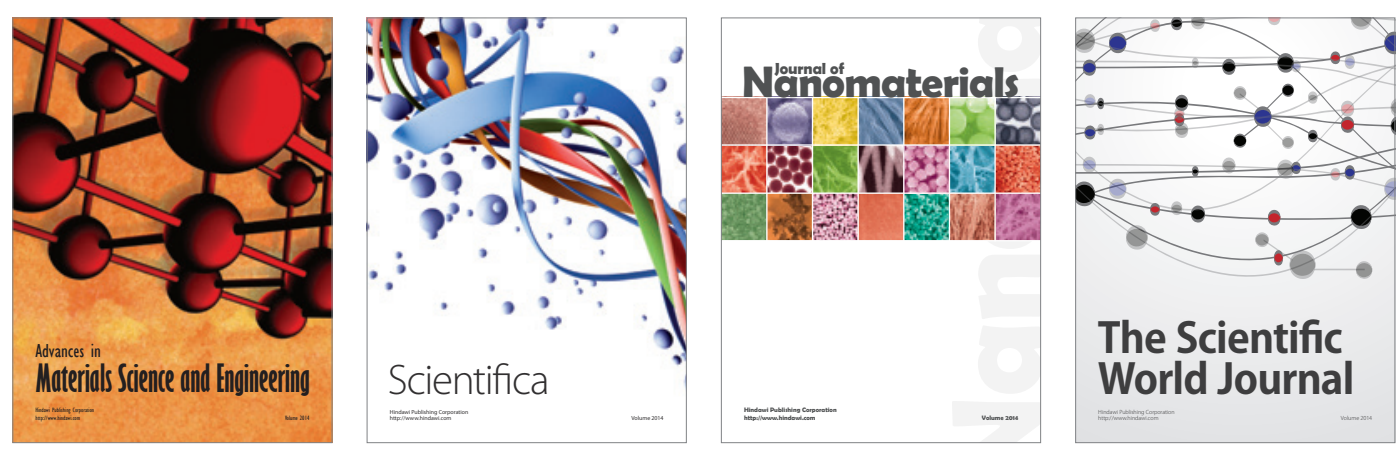

\section{The Scientific World Journal}
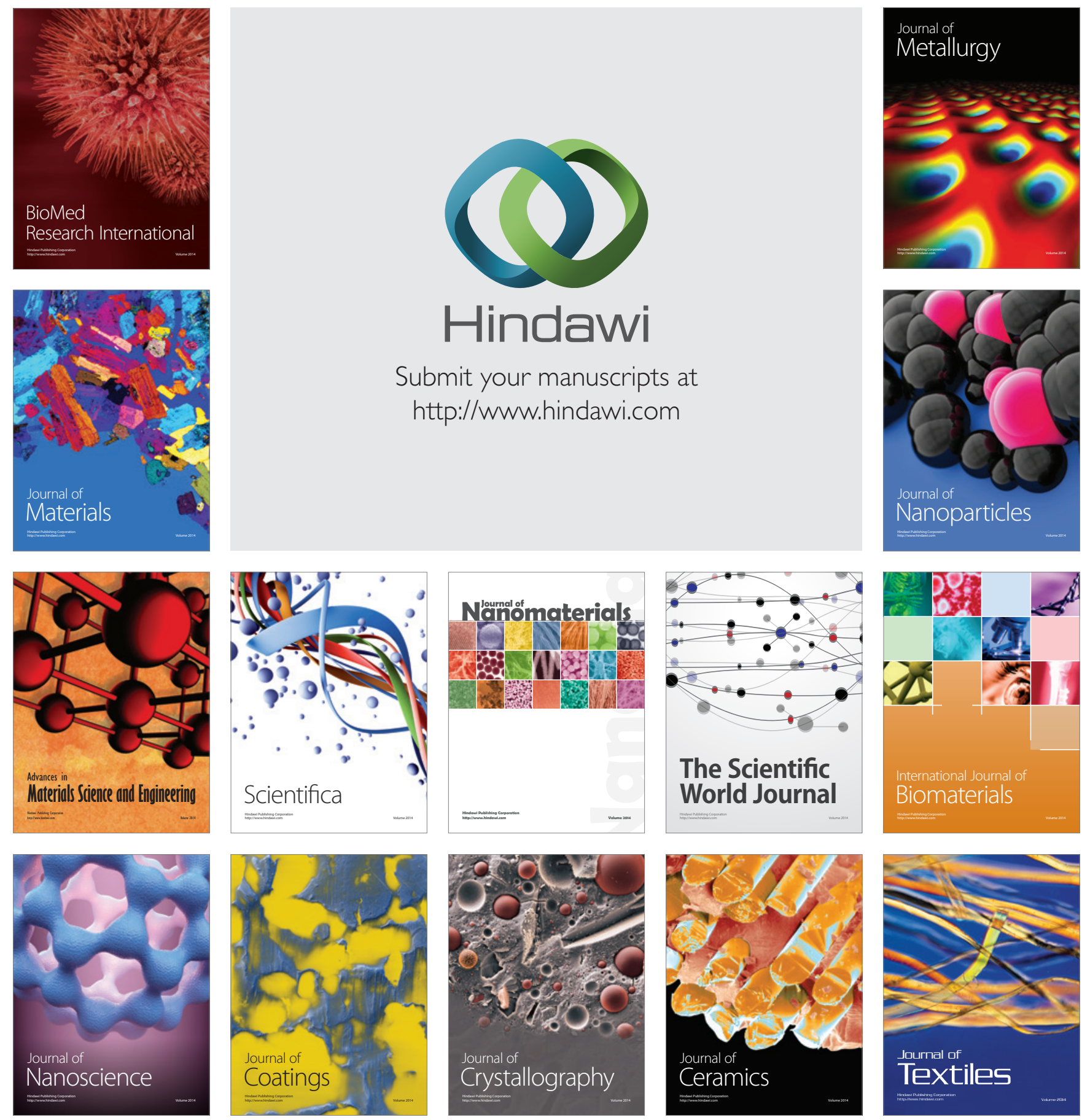